\title{
Exact and moment equation modeling of electron transport in submicron structures
}

\author{
Bernard J.Geurts, a) Maziar Nekovee, ${ }^{\text {) }}$ Henk M. J. Boots, and Martin F. H. Schuurmans \\ Philips Research Laboratories, P.O. Box 80 000, 5600 JA Eindhoven, The Netherlands
}

(Received 22 May 1991; accepted for publication 8 July 1991)

\begin{abstract}
We compare $I-V$ characteristics of a semiconducting submicron $n^{+} n n^{+}$diode as predicted by extended moment equation approximations to those obtained from the solution of the corresponding Boltzmann equation. All lower order models fail in the predominantly ballistic regime. Moreover, the conductance is inadequately predicted by these models, even in nonballistic cases due to the high build-in electric fields.
\end{abstract}

The ongoing size reduction of semiconductor devices prompts the need for descriptions extending the drift-diffusion approach. ${ }^{1}$ We compare $I-V$ characteristics of semiconducting submicron structures obtained from a systematic hierarchy of extended moment equations to those predicted by the corresponding Boltzmann equation. ${ }^{2}$ Transport of electrons in such structures is characterized by both hot and ballistic electrons as well as large gradients in e.g., the electron density and the electric field..$^{3-5}$ Further reduction of typical sizes and the use of high-mobility materials cause these effects to become even more pronounced. We study a GaAs-based $n^{+} n n^{+}$diode numerically and show that all lower order moment models give inadequate predictions of e.g., the diode conductance, particularly in the ballistic regime. For precise results one has to resort to solving the full Boltzmann equation, either by the method of characteristics ${ }^{5,6}$ or through direct Monte Carlo simulations. ${ }^{7}$ Increasing the truncation order of the moment models does not lead to a significant extension of the parameter region in which these models can be usefully applied. It is not possible to treat hallistic transport phenomena with a low order system of moment equations.

First, we introduce the systematic hierarchy of moment models and describe the procedure to obtain the closing relations. ${ }^{3,4}$ Then the predicted $I-V$ characteristics are compared with the Boltzmann equation results as a function of what we call the "ballisticity" in the system. We define this quantity as $B \equiv\left(\tau v_{d, \max }\right) / d$, where $\tau$ is the relaxation time of the collision process, $v_{d \text {, max }}$ the maximal drift velocity, as predicted by the moment models, in the diode, and $d$ the width of the $n$ region in the diode.

The stationary Boltzmann equation governs the electron distribution function $f(r, v)$ and in one spatial and velocity dimension reads ${ }^{5}$

$$
\left[v \partial_{r}-E(r) \partial_{v}\right] f(r, v)=-\frac{1}{\tau}\left[f(r, v)-f_{0}(r, v)\right],
$$

where we adopt the parabolic band approximation and assume a constant relaxation time approximation for the collision term. We use scaled variables; position $r$ and velocity

\footnotetext{
To whom correspondence should be addressed, present address: Twente University, Department of Applied Mathematics, P.O. Box 217, 7500 AE Enschede, The Netherlands.

b) Present address: Theoretical Physics 1, Katholieke Universiteit Nijmegen, Toernooiveld, 6520 ED Nijmegen, The Netherlands.
}

$v$ are measured in units $r_{0}$ (the Debye length) and $v_{0}$ (the thermal velocity), respectively, where

$$
r_{0}=\left(\frac{\epsilon k_{B} T_{0}}{e^{2} M_{\text {ref }}}\right)^{1 / 2} ; m^{*} v_{2}^{0}=k_{B} T_{0}
$$

in which $k_{B}$ is Boltzmann's constant, $T_{0}$ the lattice temperature, $\epsilon$ the permittivity, $e$ the unit of charge, $m^{*}$ the effective mass, and $M_{\text {ref }}$ a reference particle density in the system. $f_{0}$ is the distribution function describing local equilibrium, and both $f$ and $f_{0}$ are in units $M_{\text {ref }} / v_{0}$. The electric field $E$ is measured in units $E_{0} \equiv\left(m^{*} v_{0}^{2}\right) /\left(e r_{0}\right)$, and is determined by Poisson's equation which can be expressed in the above notation as:

$$
\partial_{r r} \Psi(r)=M_{0}(r)-C(r) ; E=-\partial_{r} \Psi,
$$

where $C(r)$ describes the doping profile and $M_{0}$ the particle density, in units $M_{\text {ref }}$. An $n^{+} n n^{+}$diode is represented by a doping profile $C(r)=C_{-}$in the $n$ region and $C_{+}\left(\equiv M_{\text {ref }}\right.$ ) in the $n^{+}$regions (typically $C_{-} \ll C_{+}$).

Physically relevant information is usually expressed in terms of velocity moments of the distribution function; the $m$ th moment $M_{m}$ is defined as $M_{m} \equiv\left\langle v^{m}\right\rangle$. The set of moments $\left\{M_{m}(r)\right\}$ obeys

$$
d_{,} M_{m+1}+m E M_{m-1}=-\frac{1}{\tau}\left\{M_{m}-M_{m, 0}\right\}
$$

We use the local equilibrium distribution

$$
f_{0}(r, v) \equiv \frac{M_{0}(r)}{\sqrt{2 \pi}} \exp \left(-\frac{v^{2}}{2}\right)
$$

for which the reference moments $\left\{\boldsymbol{M}_{m, 0}\right\}$ can readily be evaluated. The evolution of the $m$ th moment is governed in part by the spatial derivative of the $(m+1)$ th moment. A closing relation must be postulated expressing a higher order moment in terms of lower order moments thus rendering the Eqs. (4) finite and open for numerical treatment.

A systematic class of closing relations is generated by truncating an expansion of the distribution function $f$. Consider the following expansion of $f^{2,4}$

$$
f(r, v)=\frac{e^{-\xi^{2} / 2}}{\sqrt{2 \pi}} \sum_{j=0}^{\infty} f_{j}(r) \mathrm{he}_{j}(\xi),
$$

where $\left\{\right.$ he $\left._{j}\right\}$ are Hermite polynomials and $\xi \equiv\left(v-v_{1}(r)\right) / v_{2}(r)$. Thus, we expand $f$ around a "Max- 


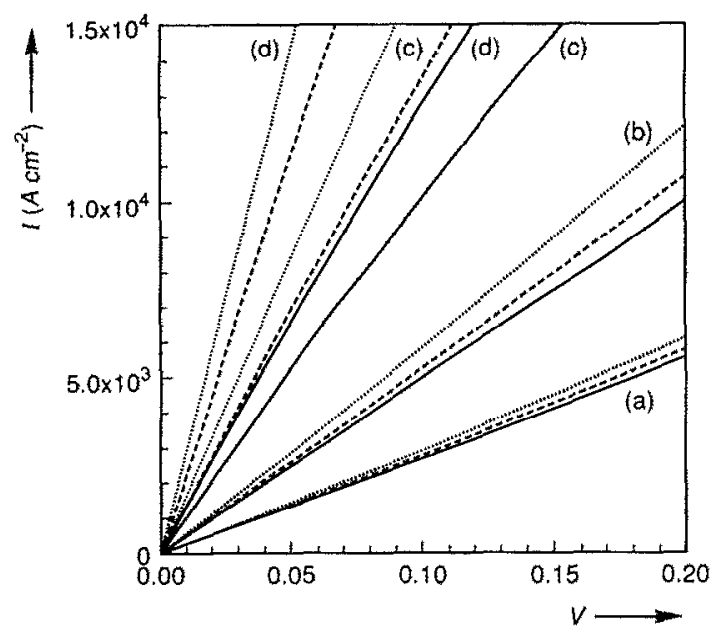

FIG. 1. Comparison of $I-V$ characteristics as predicted by the Boltzmann equation (full lines), drift diffusion (dotted lines), and four moment model (dashed lines) for a structure with a total length of $4.4 \times 10^{-6} \mathrm{~m}$ and a width of the $n$ region of $0.4 \times 10^{-6} \mathrm{~m}$. Results are shown for $\tau=5 \times 10^{-14} \mathrm{~s}$ (case a) $\tau=1 \times 10^{-13} \mathrm{~s}$ (case b) $\tau=2.9 \times 10^{-13} \mathrm{~s}$ (case c) and $\tau=5 \times 10^{-13}$ (case d). We used a lattice temperature $T_{0}=300 \mathrm{~K}$, a permittivity $\epsilon_{r}=12.5$, and an effective mass $m^{*}=0.069 \mathrm{me}_{\mathrm{e}}$ ( $m_{e}$ the electron rest mass). Finally, we used doping parameters $C_{+}=10^{24} \mathrm{~m}^{-3}$ and $C_{-}=2 \times 10^{21} \mathrm{~m}^{-3}$.

wellian" distribution with the option to include a suitably transformed velocity, viz. the introduction of $v_{1}$ and $v_{2}$. With a judicious choice of $v_{1}$ and $v_{2}$ the expansion functions $f_{1}$ and/or $f_{2}$ can be made zero and the accuracy of a truncating approximant to $f$ improved without increasing the truncation order. The expansion functions $\left\{f_{f}(r)\right\}$ can be expressed as linear combinations of the moments $\left\{\boldsymbol{M}_{m}\right\}$; $m=0,1, \ldots, j$ and the functions $v_{1}$ and $v_{2}$. Hence, truncating this expansion at order $n$ by putting $f_{n}=0$ renders a closing relation between $M_{n}$ and $\left(M_{j}\right) ; j=0,1, \ldots, n-1$. We consider moment models with order $n=4$.

Putting $v_{1}=0$ and $v_{2}=1$ implies expanding $f$ around a pure Maxwellian distribution and results in the linear closing relation $M_{4}=6 M_{2}-3 M_{0}$. Taking $v_{1}=M_{1} / M_{0}$ renders $f_{1} \equiv 0$ and corresponds to an expansion of $f$ around a "drifted Maxwellian," where the velocity is corrected with the drift velocity of the electrons. The closing relation is now nonlinear and can be written as

$$
M_{4}=4 v_{1} M_{3}+6\left(v_{2}^{2}-v_{1}^{2}\right) M_{2}-3\left[2 v_{2}^{4}-\left(v_{2}^{2}-v_{1}^{2}\right)^{2}\right] M_{0} \text {. }
$$

The function $v_{2}$ can in addition be used to render $f_{2} \equiv 0$ by taking $v_{2}$ equal to the root of the electron temperature relative to the lattice temperature. This corresponds to expanding $f$ around a "scaled and drifted Maxwellian," i.e., the velocity is corrected with the drift velocity and scaled with the local temperature. In case $n=2$, corresponding to the drift-diffusion model, expansion around a pure Maxwellian yields $M_{2}=M_{0}$ whereas an expansion around a drifted Maxwellian gives the nonlinear closing relation $M_{2}=\left(v_{1}^{2}+1\right) M_{0}$.

In Fig. 1 we show the $I-V$ characteristics of the moment models and the corresponding Boltzmann equation results for a typical $n^{+} n n^{+}$-diode. Results are shown for

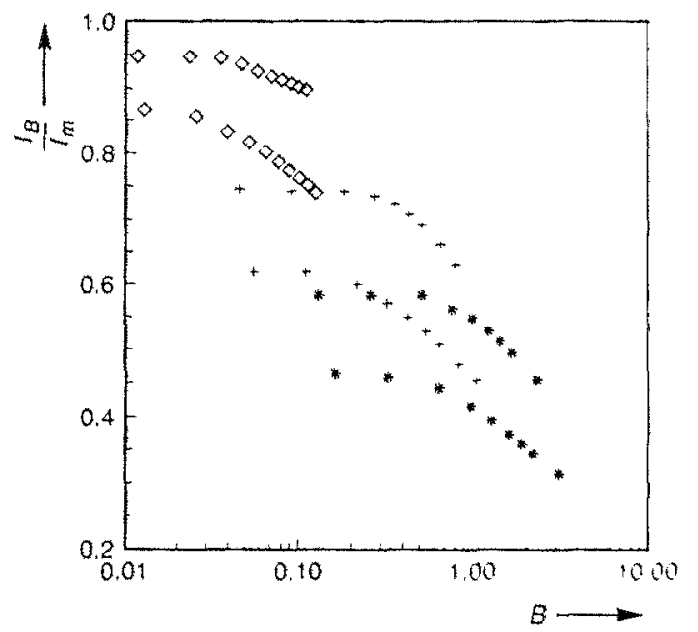

FIG. 2. Plot of $I_{B} / I_{m}$ as a function of the "ballisticity" parameter $B$ for the same parameters as in Fig. 1 . Here case (b) is represented by "diamonds"; case (c) by "plusses" and case (d) by "crosses". At each $T$ value two curves are shown; the lower presents the drift-diffusion results and the upper corresponds to the four moment results.

the drift diffusion and four moment models with linear closing relations; see also Ref. 2 . In regions where $\tau$ is small, i.e., in the nonballistic regime, all models give almost equally good results for the current as well as for the conductance of the diode, whereas in strong ballistic situations all models yield diode conductances and currents that deviate considerably from the Boltzmann results. Increasing the truncation order from two (drift diffusion) to four extends the $\tau$ region in which the deviations are less than (say) $10 \%$. However, the conductance in the strongly ballistic regime is never adequately calculated from the moment equations. The $I-V$ predictions of the "linear" and "nonlinear" moment models turn out to agree quite well with each other in the entire parameter region studied and hence the later results are not shown here. The hydrodynamic model ${ }^{8}$ was also studied. It gives adequate results for relatively low relaxation times and applied voltages. At higher relaxation times it fails to give any predictions due to instabilities related to drift velocity approaching the thermal velocity, ${ }^{9,10}$

In order to further illucidate the effects of the "ballisticity" $(B)$ in the structure we have plotted the ratio between the Boltzmann current $\left(I_{B}\right)$ and the corresponding moment current $\left(I_{m}\right)$ as a function of $B$ in Fig. 2. An increase in $\tau$ and/or a decrease in $d$ favors ballistic effects. The choice of $B$ to measure "ballisticity" in the system clearly reflects this. As $B$ is increased, i.e., ballistic effects are amplified, the agreement between the moment models and the Boltzmann model becomes less. Figure 2 shows that also the relative error in the current increases with $B$ and $\tau$. Moreover, all models give such relative error even when the applied voltage tends to zero due to the high build-in potential found in these structures. Large electric fields generated near doping steps cause locally far from equilibrium situations to be reached already at small applied voltages. Finally, we observe that at high ballisticity $\left(I_{B} / I_{m}\right) \sim \log (B)$, for all moment models. 
Our evaluations for the $n^{+} n n^{+}$diode indicate that strong ballistic effects cannot be adequately described with any low-order moment model. Of course, the use of the relaxation time approximation for the collision integral in the Boltzmann equation, implies an exaggeration of ballistic effects. ${ }^{5.11}$ However, we anticipate that even when using more realistic scattering terms, the same sort of discrepancies will be found in predominantly ballistic situations. In essence, the distribution function develops a sharp highvelocity ballistic peak in far from equilibrium situations, next to a "bell"-shaped near equilibrium distribution at lower velocities within the structure. ${ }^{5,6,11}$ This intricate structure can no longer be represented by any small number of moments, e.g., a single typical velocity and temperature. Conversely, in the nonballistic regime such a highvelocity peak is absent and the essential structure of the distribution function can quite well be captured with a small number of typical scales/moments.

The authors have greatly appreciated discussions with Professor G. Schön at Delft University and T. Portengen at UCSD.

'R. K. Cook and J. Frey, IEEE ED-29, 970 (1982); C. T. Wang, Solid State Electron. 28, 783 (1985); W. Hänsch and M. Miura-Mattausch, J. Appl. Phys. 60, 650 (1986); E. Schöll and W. Quade, J. Phys. C 20,
L871 (1987); B. Meinerzhagen and W. L. Engl, IEEE, ED-35, 689 (1988); E. M. Azoff, J. Appl. Phys. 64, 2439 (1988); K. Blotekjaer, IEEE, ED-17, 38 (1970); R. Stratton, Phys. Rev. 126, 2002 (1962).

${ }^{2}$ M. Nekovee, B. J. Geurts, H. M. J. Boots, and M. F. H. Schuurmans, Failure of Extended Moment Equation Approaches to Describe Ballistic Transport in Submicron Structures, Philips Research Laboratories (1991) (unpublished).

${ }^{3}$ A. Bringer and G. Schön, J. Appl. Phys. 64, 2447 (1988).

${ }^{4}$ T. Portengen, H. M. J. Boots, and M. F. H. Schuurmans, J. Appl. Phys. 68, 2817 (1990).

${ }^{5}$ H. U. Baranger and J. W. Wilkins, Phys. Rev. B 36, 1487 (1987).

${ }^{6}$ B. J. Geurts, Characteristic Method for Solving the Spatially Inhomogeneous Boltzmann Equation, Philips Research Laboratories (1991) (unpublished).

${ }^{7}$ P. J. Price, in Semiconductors and Semimetals, edited by R. K. Willardson and A. C. Beer 14, 249 (Academic, New York, 1979); C. Jacobini and L. Reggiani, Rev. Mod. Phys. 55, 645 (1983); C. Moglestue, IEEE Trans. CAD 5, 326 (1986); K. Hess, Advanced Theory of Semiconductor Devices (Prentice Hall, Englewood Cliffs, 1988); M. V. Fischetti and S. E. Laux, Phys. Rev. B 38, 9721 (1988); E. Sangiorgi, B. Riccó, and F. Venturi, IEEE Trans, CAD 7, 259 (1988).

${ }^{8}$ G. Baccarani and M. R. Wordeman, Solid State Electron. 28, 407 (1985); We use $\tau=$ constant in all moment equations and a Wiedeman-Franz law in the Fourier closing relation.

${ }^{9}$ B. J. Geurts, An Extended Scharfetter Gummel Scheme for High Order Moment Equations, Philips Research Laboratories (1991) (unpublished).

${ }^{10}$ C. L. Gardner, J. W. Jerome, and D. J. Rose, IEEE Trans, CAD-8, 501 (1989).

${ }^{11}$ B. J. Geurts, J. Phys. C (in press). 